\title{
AN INNOVATIVE LOW COST BONE DENSITOMETER BASED ON CONVENTIONAL X-RAY FACILITY
}

\author{
Jubaid Abdul Qayyum ${ }^{1}$, Md. Masum Howlader ${ }^{1}$, Md. Tamzeed-Al-Alam ${ }^{1}$, \\ Md. Saiful Islam ${ }^{1}$, Tahmid Latif ${ }^{1}$, and K Siddique-e Rabbani \\ Department of Biomedical Physics \& Technology, University of Dhaka, Dhaka-1000, Bangladesh \\ email: jubaidaq@yahoo.com, unique_masum@live.com, rabbani@univdhaka.edu
}

\begin{abstract}
Osteoporosis or bone loss affects many people, particularly the aged women, and leads to disabling bone fractures. An early diagnosis could provide preventive management. However, available bone densitometry equipment are very expensive and not available widely in the Third World. The present work presents the design \& development of an innovative low cost bone densitometer based on conventional X-ray equipment available widely. The main innovation is the incorporation of a stack of aluminium with varying thickness placed beside the limb of a patient while taking an X-ray image. Then the optical density of the bone area in the developed film is compared to that from the aluminium stack, which eliminates variations due to all other processing factors, and gives a measure of the bone density. A low cost device has been developed to measure the optical density of the X-ray film over a circular area of about $1 \mathrm{~cm}$ to give an average reading, which is more appropriate than spot metering in this application. Preliminary measurements of a few human subjects using this equipment clearly indicate the differences obtained due to expected osteoporosis. Calibration with standard bone densitometry equipment could provide values in standard units. This low cost innovative method and equipment appears to provide a low cost alternative to the diagnosis of osteoporosis.
\end{abstract}

Keywords: Bone densitometer, X-ray equipment, Osteoporosis

\section{INTRODUCTION}

Bone density reduction or osteoporosis is a disabling condition, particularly at old age, and for women, more so for those suffering from malnutrition. Bone consists of a matrix of collagen fibres providing a substructure into which is embedded hydroxyapatite, or minerals composed mainly of calcium and phosphate compounds. In osteoporosis, the hydroxyapatite wears off and the bone becomes brittle, resulting in undesirable fractures. The degeneration is indicated in the micrographic depiction of bone structure in Fig.1 [1]. In an X-ray investigation, the amount of X-ray that is attenuated by bone depends on its density, therefore, an osteoporotic bone will provide less attenuation. Since an X-ray film gives a negative image, an osteoporotic bone will give a darker image compared to a normal bone. The pictures shown in Fig. 2 [2] depict this information clearly where a darker image is produced for an osteoporotic bone. This condition of osteoporosis needs to be diagnosed early for predicting fracture risks, and for medical intervention, if required. Specialised X-ray equipment is available for such diagnosis [3], but is expensive and not widely available in Third World countries. Since conventional X-ray equipment to provide images as shown above is available in most Third World hospitals, it was thought that an improvisation to obtain quantitative values for bone density using these conventional equipment would be worthwhile. The present paper presents this work, from basic concepts to a practical prototype which could at least show some differences in densities of subjects expected to have different conditions including osteoporosis.

\footnotetext{
${ }^{1}$ Islamic University of Technology, Gazipur, Bangladesh
} 


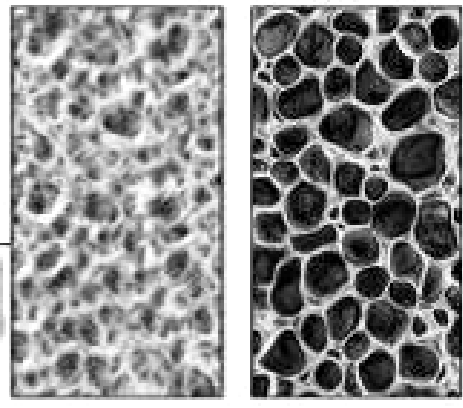

Fig. 1: Micrographic depiction of normal bone structure (left) and osteoporotic bone (right).
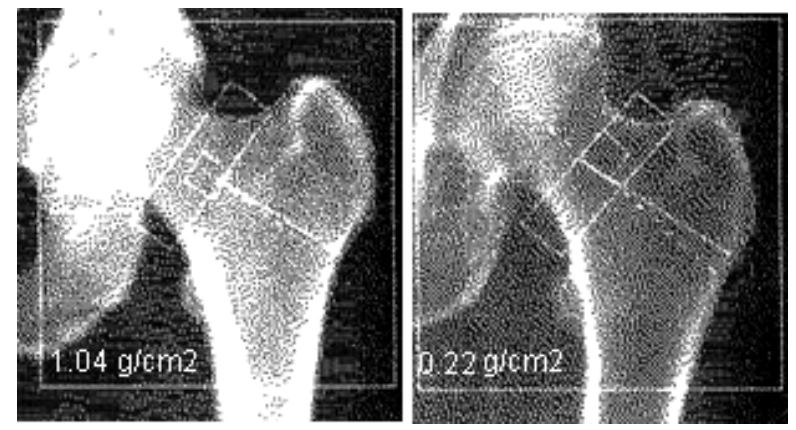

Fig. 2: X-ray absorption images of bone. Brighter image on left indicate higher bone density (normal) compared to that on the right (osteoporotic).

\section{METHODS}

\subsection{Basic concepts}

The idea was to assess the bone density of a subject using conventional X-ray equipment in which a transmission image is obtained on an X-ray film. The optical density at the desired bone position may be assessed using a digital camera and software to evaluate individual pixel values. The same may be performed using a low cost scanner too. Alternatively a very low cost arrangement may be built up using a light source based on a light emitting diode (LED) and a photo detector to evaluate the average optical density of a localized zone in the X-ray film. It needs appropriate optical and electronic design which may be achieved at a small cost. The present work was taken up to design and develop this low cost option.

2.1.1 Elimination of uncertainties: However, the optical density of a developed X-ray film depends on a variety of factors such as film speed, exposure time, development time, concentration of developing chemicals, temperature, etc. besides the desired bone density. Therefore, to correlate the optical density at the bone to its physiological condition all the other variables need to be eliminated. The innovation in the present work to this end was simply to have an object with a constant optical absorbance to X-ray, to be placed beside the body segment to be imaged during taking of the X-ray image. During the measurement of optical density of the bone segment, the density of this object would act as a reference, thus eliminating all the other variables as required. In the present work a stack of aluminium strips providing different thicknesses was used to provide this calibration. However, the optical density of the images produced by Aluminium also depends on the X-ray tube voltage [4], since the spectrum varies under such variations. Therefore, either the X-ray voltage should be kept constant in all the tests, or, a correction factor should be developed to take care of differing X-ray tube voltage.

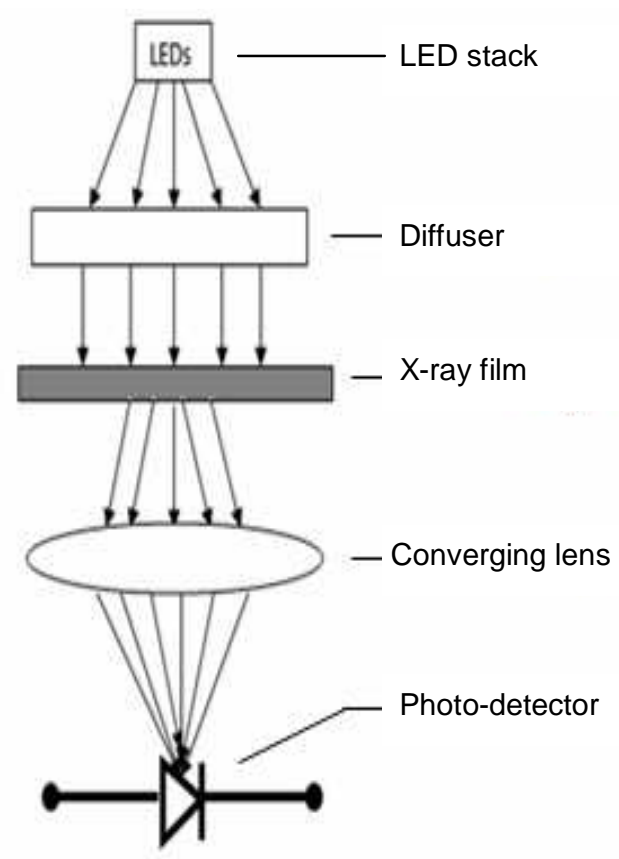

Fig 3: Overview of the designed set up 


\subsection{Design and fabrication of optical equipment}

The design consists of a light source providing a uniform illumination over a certain area where the X-ray film is to be placed. The area of illumination was chosen as a circle of diameter $1 \mathrm{~cm}$. This was chosen against a spot metering system because point to point density may vary widely between neighbouring points on the film as may be envisaged from the pictures in Figs. 1 and 2. The use of this $1 \mathrm{~cm}$ diameter aperture would give an average density which is expected to be a better indicator minimizing the chances of erroneous interpretation. However, in a finished device, a choice of aperture diameters may be provided to suit a particular region of bone to be assessed.

The transmitted light was concentrated onto a photo-diode using a convex lens. The output current from the photo-diode, which is proportional to the intensity of incident light, is processed using appropriately designed electronic circuitry to give a voltage value which can be read using a digital voltmeter. This voltage value would be proportional to the transmitted light through the X-ray film. Since a dense bone gives a lighter X-ray image, it would give

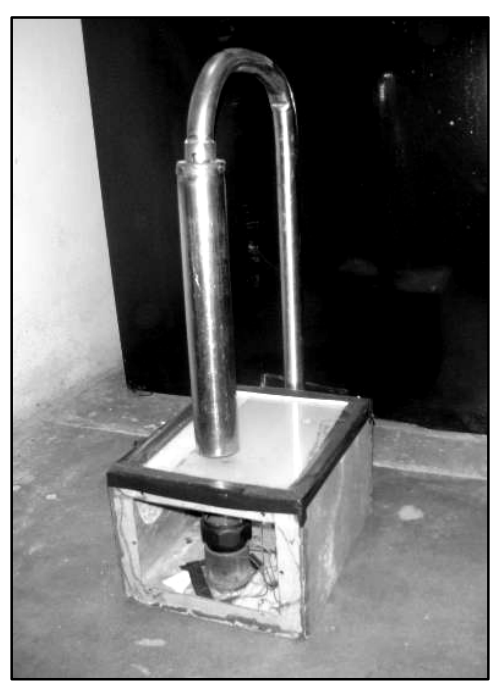

Fig. 4: The first prototype a greater output voltage value compared to that for an osteoporotic bone. Fig. 3 shows a schematic of the whole design while Fig. 4 shows a picture of the finished prototype. A detailed description of the design is given below.

2.2.1 Light Source: Two alternative options were considered, using a cluster of red LEDs, or using a low cost solid state LASER available in the market as a pointer for lecture presentations. Since the LASER has a small beam diameter, it had to be spread out using a concave lens to obtain the desired aperture of $1 \mathrm{~cm}$ diameter. However, this spread out beam appeared to have dark spots and non-uniform illumination. Therefore, it was discarded and a system based on LEDs was developed. A cluster of red ultra-bright LED's provided an extended source the light pointing downwards which was then diffused using a milk white acrylic plastic sheet. A collimator was used to obtain uniform lighting over a circular area of diameter $1 \mathrm{~cm}$, where the X-ray film is to be placed for measurement. The LEDs, diffuser and the collimator were mounted inside a hollow metallic tube as shown in Fig.5.

2.2.2 Light collector: A converging lens is used to produce a real and sharp image on the sensitive portion of the photodetector as shown in Fig.3. The aperture of the photodetector had a diameter of about $2.5 \mathrm{~mm}$. Therefore to get the whole of the $1 \mathrm{~cm}$ diameter of the object a magnification of 0.25 was necessary. This means that the object distance, i.e., the distance from the X-ray film and the lens should be four times as great as the image distance. Again, the more is the object distance the less light from the object will be gathered by the lens. Therefore, a small focal length of the lens was desired. This was also desirable in order to keep the equipment within a reasonable height. Lenses with very small focal lengths were not readily available, so two convex lenses were combined to get a small focal length of about $3.5 \mathrm{~cm}$.

2.2.3 Light Detector: A silicon photodiode was chosen as the light detector. Current output from a photodiode has excellent linearity characteristics with incident light. Therefore, this was

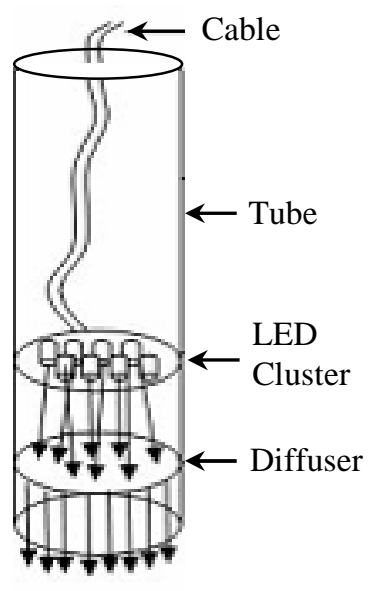

Fig.5: Schematic diagram showing internal arrangement of the light source. 
very suitable for an analogue measurement of the intensity of light incident on the detector. A current to voltage converter circuit using an operational amplifier was designed which gave a voltage output that could be measured using a digital voltmeter. The transfer gain could be adjusted by adjusting a feedback resistor in the circuit.

\section{RESULTS AND DISCUSSIONS}

\subsection{Calibration}

As mentioned above, a stack of aluminium strips giving various thicknesses provided the means to calibrate the developed X-ray film. Fig.6 shows a photograph of the stack made up of 12 aluminum strips each $1 \mathrm{~mm}$ thick and of gradually increasing length. These were held into place using black plastic adhesive tape, thus providing effective aluminium thicknesses of $1 \mathrm{~mm}$ to $12 \mathrm{~mm}$ in steps of $1 \mathrm{~mm}$. This aluminium stack will be useful in giving a reference for all measurements for bone density on the X-ray films, eliminating variation in film densities due to factors except the bone density, as discussed before.

An X-ray image obtained by placing this stack on an X-ray film in a conventional diagnostic X-ray equipment is shown in Fig.7. It shows the variation in exposed film densities, dark on the left side for the thinnest part, to light on the right side corresponding to the thickest part of the stack. The X-ray film with the above image was measured and analysed using the developed prototype of the bone densitometer. The output voltage values obtained from this measurement is plotted in Fig. 8 corresponding to different thicknesses of aluminium. It should be noted that some output voltage value was obtained even for the darkest part of the X-ray film, so this was subtracted from each of the measured output values shown to obtain the figure. The plotted points were fitted using an exponential curve in EXCEL which shows a very good agreement.

To see if this behaviour agrees with what is expected from the basics of X-ray absorption the following argument may be put forward. If $I_{o}$ is the incident beam intensity and $\mathrm{I}$ is the transmitted intensity after going through a thickness $t$ of aluminium, the relationship would be given by [5],

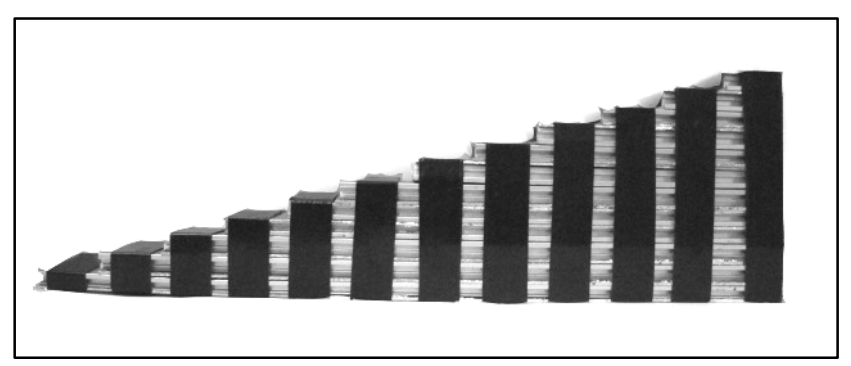

Fig 6: Photograph of Aluminum strips of different lengths stacked onto one another and strapped using black tape to provide thickness from $1 \mathrm{~mm}$ to $12 \mathrm{~mm}$

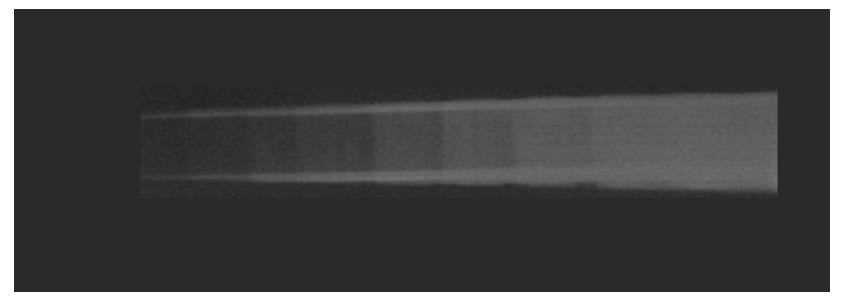

Fig 7: X-ray image of Aluminum strip arrangement in Fig 6

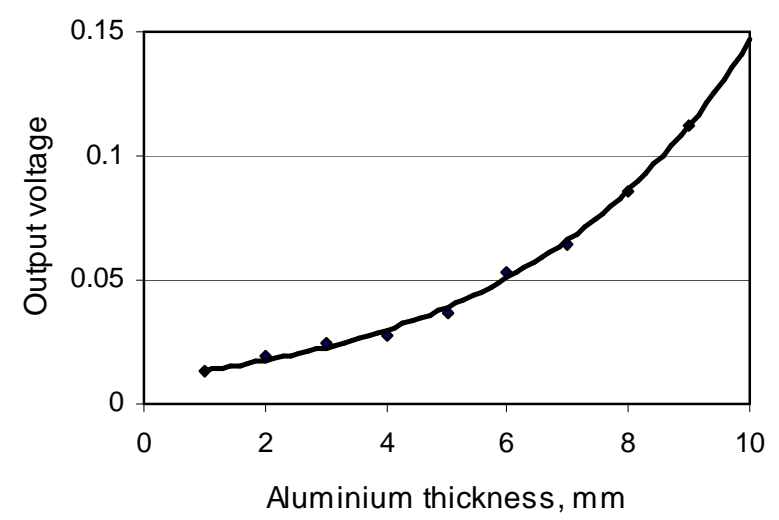

Fig 8: Output voltage of the bone densitometer with density of X-ray film shown in Fig.7. 


$$
I=I_{0} e^{-a t}
$$

where $a$ is the absorption constant

The exposed and developed X-ray film gives a negative or inverse image; it has a lighter shade for less intensity of incident X-ray, and has a dark shade where the intensity is high. The optical device developed in the present work gives more output voltage for a lighter shade of the X-ray film, i.e., where the X-ray beam intensity is low. Therefore the output voltage of the device is expected to be inversely proportional to the X-ray intensity. Then the voltage output will be given by,

$$
V=V_{0} e^{a t},
$$

which shows that the output voltage will increase exponentially with the thickness of aluminium, as obtained experimentally.

\subsection{Measurement on human subjects}

As a preliminary test for the developed equipment four human subjects were studied. Two of them were young healthy males, 23 years of age. One subject of 35 years had a handicapped foot while the fourth subject was old, 60 years of age. For the first three, X-ray images were obtained in the region of the ankle, while for the fourth subject, the shoulder region was imaged. The aluminium strips were placed by the side of the subject, within the film exposure area when taking the X-ray image. Fig. 9 shows that obtained from subject 1 . The white circle on the ankle image is the region for which the X-ray film density was measured giving a corrected output voltage as given in Table 1. Then the X-ray image of the aluminium strips was moved under the measurement aperture till the same output voltage is obtained. From the thickness of the aluminium strips in that location an equivalent aluminium thickness, in $\mathrm{mm}$, was obtained and is shown in Table 1 as well. For subject 2 , the measured output voltage was beyond the values obtained using the aluminium strips. In this case, the equivalent thickness was extrapolated from the exponential equation fitted to the graph in Fig.8. For subjects 3 and 4, values could be obtained from the image directly as for subject 1 . The equivalent thickness of aluminium can later be transformed into a measure for bone density in comparison with standard bone density equipment. Because of the nonavailability of such equipment the present work could not give any absolute values of bone density.

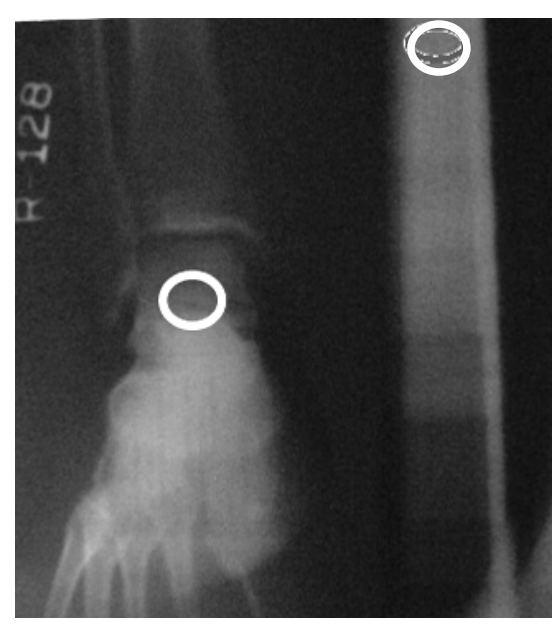

Fig.9: X-ray image of ankle of subject 1 beside that of aluminium strips. The circled areas on both the ankle and the aluminium strip image had equal density values.

Table 1 also shows a good agreement with expected values from human subjects on a qualitative basis. The ankles of the two normal young male subjects both have very close and high values, aluminium equivalent thickness of $11 \mathrm{~mm}$ and $12 \mathrm{~mm}$ respectively. The person with a handicapped foot had only $3 \mathrm{~mm}$ equivalent thickness at the ankle. It is likely that lack of use made his bone osteoporotic at that location. The female subject at the age of 60 is likely to be osteoporotic, which is also borne out by the very low equivalent aluminium thickness value. Of course, the location is different here, the shoulder, where some difference is likely. However, the very low value obtained may indicate the presence of osteoporosis. 
Table 1: Bone density in equivalent aluminium thickness observed on human subjects. The values match those expected qualitatively.

\begin{tabular}{|c|c|c|c|c|c|c|}
\hline $\begin{array}{c}\text { Subject } \\
\text { code }\end{array}$ & Sex & $\begin{array}{c}\text { Age } \\
\text { yrs }\end{array}$ & Physical condition & $\begin{array}{c}\text { Location of } \\
\text { exposure }\end{array}$ & $\begin{array}{c}\text { Corrected } \\
\text { output } \\
\text { voltage } \\
\text { V }\end{array}$ & $\begin{array}{c}\text { Equivalent } \\
\text { Aluminum } \\
\text { Thickness } \\
\text { mm }\end{array}$ \\
\hline 01 & M & 23 & Healthy & Ankle & 0.153 & 11 \\
\hline 02 & $\mathrm{M}$ & 23 & Healthy & Ankle & 0.292 & 12 \\
\hline 03 & $\mathrm{M}$ & 35 & Handicapped foot & Ankle & 0.022 & 3 \\
\hline 04 & $\mathrm{~F}$ & 60 & Aged & Shoulder & 0.013 & 1 \\
\hline
\end{tabular}

\section{CONCLUSIONS}

The device presented is the first attempt at developing the new idea, in the making of a low cost bone densitometer for the diagnosis of osteoporosis using conventional X-ray devices available widely in the Third World. The calibration curve shown in Fig.8 has a very good agreement with an exponential fit. This is also expected from the basic principles as shown.

It would be very useful if the measurements can be compared with that obtained from a standard bone densitometer equipment, giving bone density in absolute units. However, the 'equivalent aluminium thickness' used in the present work may provide a useful unit for practical purposes, for equipment based on the present innovation. This will need a statistical study of many patients, normal and diagnosed for osteoporosis. Also in vitro measurements with dead bone may be carried out to obtain absolute density values.

In an actual measurement, interposed soft tissue will also contribute to the X-ray image intensity and the output obtained. In order to compensate for such soft tissue, X-ray image density at a point having similar thickness of soft tissue only can be subtracted. However, in regions like the heel, the thickness of soft tissue is negligible and such compensation may not be necessary.

Further improvement of the device is necessary before it can be used with confidence for diagnosis, but this work shows that this new idea is feasible, and will have widespread application in the Third World in the measurement of osteoporosis.

\section{REFERENCES}

1. http://www.radiologyinfo.org/en/info.cfm?pg=dexa

2. http://courses.washington.edu/bonephys/opbmd.html

3. http://en.wikipedia.org/wiki/Dual-energy_X-ray_absorptiometry

4. M. Begum et al, Quality control tests in some diagnostic X-ray units in Bangladesh, Bangladesh Journal of Medical Physics, Vol.4, No.1, 2011 (this issue).

5. http://en.wikipedia.org/wiki/Beer\%E2\%80\%93Lambert_law 